\title{
INTEGRACIÓN DE LOS ESTUDIOS DEL MEDIO FÍSICO \\ EN LA ORDENACIÓN DEL TERRITORIO: VALORACIÓN ECOLÓGICO-PAISAJÍSTICA DEL RÍO ALGAR \\ (ALTEA, ALICANTE)
}

J. A. Marco Molina

E. Matarredona Coll

A. Padilla Blanco

RESUMEN

Tras un análisis de las condiciones físicas que caracterizan al río Algar, Altea (Alicante), se definen y valoran unas unidades ambientales bajo distintos puntos de vista, con el fin de establecer las repercusiones y efectos de las intervenciones humanas en este espacio, así como una serie de actuaciones que reactiven este cauce en su paso por el municipio de Altea. Este trabajo se estructura en una fase analítica, de conceptuación y definición de las unidades fisiográficas y morfológicas del río, de las unidades de vegetación presentes en este ecótopo, así como de las intervenciones antropogénicas, sus repercusiones y posibles «impactos»; y, una segunda, sintética, que tiene como objetivo la caracterización de distintas zonas a tenor de su calidad ambiental. Para ello, en esta última fase se establecen las oportunas interrelaciones entre cada uno de los elementos tratados, con lo que, de la consideración tanto de aspectos relativos a las limitaciones que impone el medio (riesgos), como de la conservación de áreas con un determinado nivel de calidad ambiental, se acomete una valoración y propuesta de actuaciones encaminadas a la reactivación de este espacio.

Palabras clave: medio físico, morfología fluvial, paisaje vegetal, humanización, riesgos, calidad ambiental, ordenación territorial.

\section{ABSTRACT}

After an analysis of the physical conditions that characterize Algar river, some environmental units are defined and valued under different points of view, in order to establish the repercussions and effects of the humans interventions in this space, as well as a kind of performances that reactivate this riverbed as it's passing by the municipality of Altea. This work is structured on an analytical phase, of conceptuation and definition of the physiographic and morphological units of the river, of the present vegetation units, 
as well as of the human interventions, its repercussions and possible impacts and, a second, synthetic, that has as objective the characterization to the different zones depending of its environmental quality. Whith this objetive, in the last phase are established the logical interrelationships between each one of the discussed elements, with what, of the consideration of aspects related to the limitations that imposes the means (risks), as well as the conservation of the areas with a given level of environmental quality, it is made a valuation and proposal focus to the reactivation of this space.

Key words: physic environmental, fluvial morphology, biogeographic landscape, humanisation, hazards, environmental quality, land management.

\section{Objetivos y metodología}

Todo estudio tendente a la planificación u ordenación territorial, sea cual sea la escala de trabajo utilizada, no puede obviar el análisis de la infraestructura o soporte físico del paisaje. Los precedentes más antiguos de este tipo de estudios, al igual que la denominación de «planificación física», son los trabajos de proyectistas ambientales norteamericanos (McHargh, 1966). En este sentido, González Bernáldez señala que la introducción del conocimiento del medio físico en los estudios regionales se remonta a los dictámenes sobre la capacidad de uso del territorio, fruto de los «estudios integrados», tras la Segunda Guerra Mundial, en consonancia con el auge de la planificación física, inspirada en los Planes Hidrológicos y de Uso del Suelo Agrícola. De entre los estudios de diagnóstico, destacan los trabajos de McHargh, relativos a minimización de los impactos provocados por las intervenciones antrópicas, sintetizados en su obra Design with nature (New York, 1969).

En esta línea de trabajo se enmarcan una serie de estudios relacionados con la planificación y ordenación territorial en el ámbito alicantino, tanto a escala municipal como comarcal. En todos ellos ha ocupado un lugar destacado el tratamiento del medio físico como pieza clave para el desarrollo del esquema de trabajo, puesto que de su consideración se han desprendido medidas de tipo prospectivo encaminadas a la valoración-diagnóstico de aquellos espacios en los que deben primar actuaciones de tipo limitativo o de condicionamiento de la capacidad de aprovechamiento de ciertas áreas a tenor de su calidad ambiental, así como de la peligrosidad derivada de la naturaleza de ciertos recursos geomorfológicos.

Se utiliza una metodología o esquema de trabajo que enlaza con planteamientos similares en este tipo de trabajos (CEOTMA, 1984; GÓMEZ OREA, 1994) fundamentado en dos fases totalmente distintas, pero indefectiblemente complementarias y, al mismo tiempo, interrelacionadas y yuxtaponibles:

- La primera de estas fases, dedicada al análisis de las condiciones físicas del territorio, parte de la consideración de éste como algo constituido por la superposición o agregación de una serie de elementos, tanto abióticos como bióticos, los cuales son objeto de estudio como información básica de partida que se materializa en una cartografía temática, referida a cada uno de ellos.

- Una vez finalizada esta fase analítica, se aborda una segunda, de carácter claramente prospectivo, en la que se realiza una síntesis y valoración del complejo ecológico. Para ello, y con el ánimo de tratar todos aquellos aspectos que puedan tener un interés vital en las posteriores propuestas de zonación u ordenación territorial, se consideran una serie de unidades en las que se limitarán sus aprovechamientos, bien por la existencia de hechos físi- 
LOCALIZACIÓN DEL ÁREA DE ESTUDIO

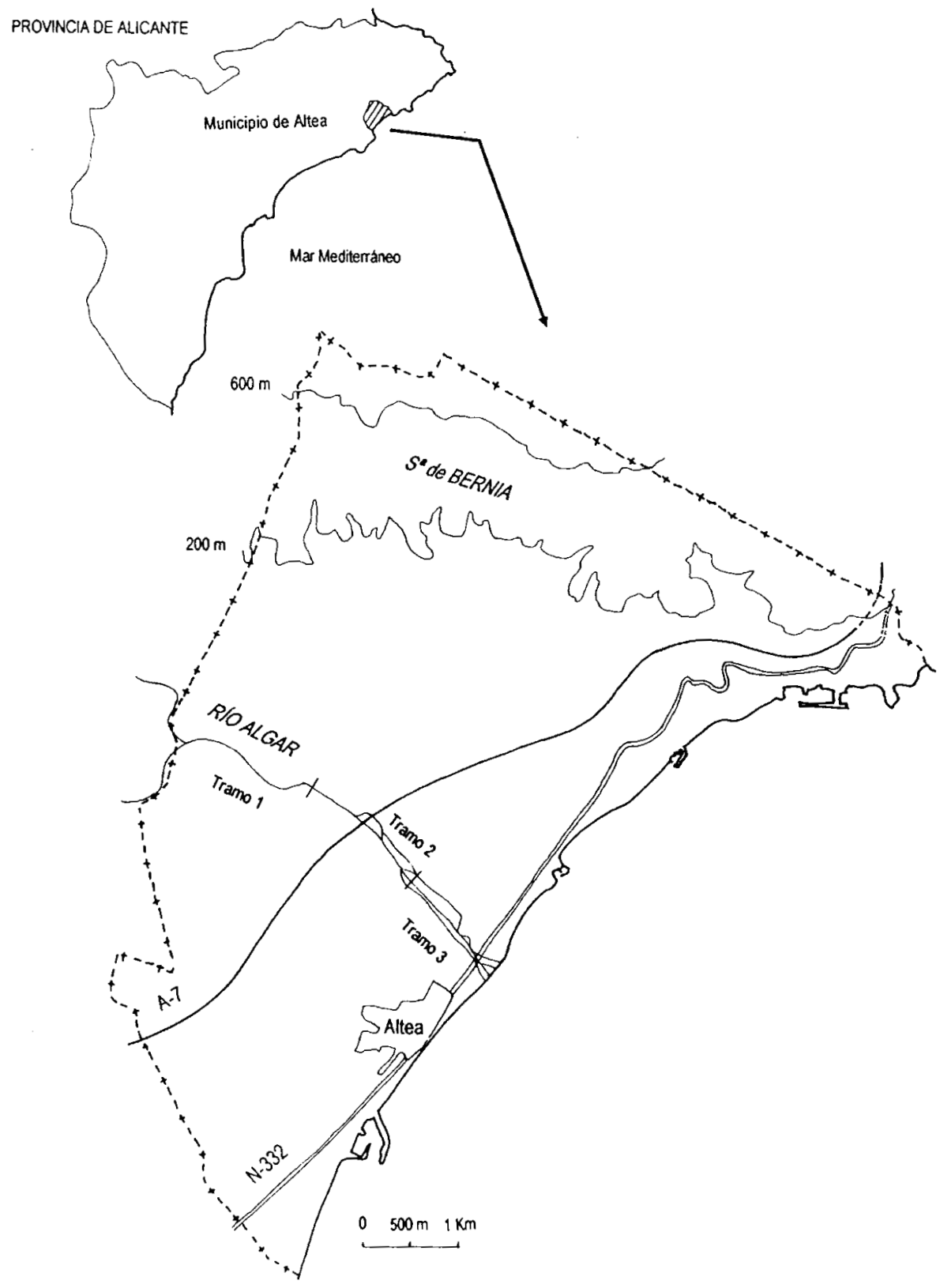

Figura 1. Localización del área de estudio. 
cos de consecuencias claramente limitativas en cuanto al uso del suelo, bien porque existan áreas de notable calidad desde un punto de vista ecológico o fisiográfico-paisajístico.

Este esquema se aplicó a escala local en un estudio de carácter global e interdisciplinar sobre el término municipal de Altea (vid. figura 1). La razón por la que se realiza un tratamiento específico del río Algar es la importancia que éste tiene a escala municipal, tanto en lo referente a cuestiones medioambientales, como a su papel como eje articulador de la organización tradicional del espacio agrario alteano. Como sector perfectamente singularizado de los espacios aledaños, es preciso remitirse a aquellos aspectos que confieren dicha singularidad al mismo, tanto en lo relativo a rasgos de orden físico, como de la interrelación entre éstos y la actuación humana.

Por estos motivos, el esquema de trabajo específico se estructura en una primera parte analítica, de conceptuación y definición de las unidades fisiográficas y morfológicas del río, así como de las formaciones o unidades de vegetación que tienen cabida en este ecótopo de acusada singularidad; además, en esta primera fase, es obligado referirse, igualmente, a las intervenciones antropogénicas, tanto si éstas se adaptan a las condiciones del medio, como si, por sus repercusiones y efectos, pueden considerarse como impactos. Por último, la segunda fase, sintética, tiene como objetivos no sólo la caracterización de las distintas unidades ambientales, sino también su valoración y relación con posibles actuaciones encaminadas a una reactivación y puesta en valor de este espacio.

\section{Fase analítica. Condiciones físico-ecológicas del territorio}

\subsection{Consideraciones previas. Red hidrográfica y morfometría}

Resulta éste uno de los apartados de mayor interés en el desarrollo del presente trabajo, por cuanto que, por una parte, nos permite señalar las principales formas derivadas de los procesos erosivos, especialmente los pasados; mientras que, por otra, son pilar básico en el reconocimiento, definición y diagnóstico de la dinámica actual, elemento clave en la definición de riesgos geomorfológicos, que se acomete en la fase prospectiva.

De origen fluvial, son las formas predominantes, de manera que la instalación de la red de avenamiento, sobre todo del Algar, es la responsable de la morfología de lomas, característica de todo el sector del diapiro, en un claro ejemplo de disección determinado por la erosión diferencial. No obstante, si este relieve de lomas lo localizamos en el afloramiento diapírico del Keüper, no podemos obviar, que también existen razones de orden estructural que, en definitiva, son las que han subordinado el comportamiento de la red de avenamiento; estos argumentos son: la remanencia en el comportamiento diapírico de los materiales del Keüper y la importancia que para la evolución geomorfológica tienen los reducidos asomos de rocas duras — rocas encajantes o infrayacentes- que, arrastradas y levantadas por el ascenso diapírico, dan verdadero sentido a la actuación de la erosión diferencial, por cuanto que son pequeños fragmentos de la cobertera sedimentaria que actúan como sellos o corazas protectoras respecto de los efectos de la erosión. Estos hechos se reflejan en un relieve alomado, a base de tossals y llomes, que destacan del orden de 40-60 metros respecto de los valles o vaguadas aledañas que confieren cierta anfractuosidad, al tiempo que singularidad, al valle como unidad de relieve.

Por otro lado, el mayor protagonismo lo acapara el propio Algar, definiendo un típico valle en artesa encajado en sus propios sedimentos (terrazas), constituyendo un cauce de fondo plano, si bien con las irregularidades condignas de un curso entrelazado (lecho braided). 
Por último, mención especial merece, el modelado de detalle que presentan los materiales del Keüper en el sector de la confluencia del Algar con el Guadalest en forma de cárcavas o abarrancamientos.

La consideración de la red hidrográfica constituye un capítulo obligado tanto en esta fase analítica inicial, como en el posterior desarrollo de la fase sintética, en la que que se integran todos aquellos aspectos del medio físico que tienen una significación especial en la definición de las distintas capacidades de acogida de cada porción del territorio. Es obligado, pues, caracterizar la cuenca vertiente del Algar con el objetivo fundamental de ponderar debidamente las posibles consecuencias morfológicas y dinámicas de su funcionamiento en momentos de acusada excepcionalidad que, en definitiva, son los que rigen las pautas de su comportamiento y, en suma, nos permiten señalar los puntos de mayor riesgo.

Para cubrir estos objetivos básicos, no obstante, es preciso realizar unas consideraciones previas, pues nadie discutirá, seriamente, que el análisis de las cuencas vertientes debe abordarse desde una óptica global; y dicha globalidad se refiere a la totalidad territorial del sistema que constituye cada cuenca vertiente, de manera que, si bien en algunas ocasiones, de cada colector, y en especial del río Algar, sólo haya representación de sus tramos más bajos, localizados en el término municipal, no podemos volver la espalda a las respectivas cabeceras, puesto que es allí donde se genera la mayor parte de los débitos, tanto líquidos como sólidos.

La cuenca del Algar, pluviométricamente, se sitúa en un bastión fronterizo entre las cuencas situadas al norte, bien regadas, y las ubicadas al sur, en las que se evidencia el efecto de sombra pluviométrica. La cabecera de ésta se incluye todavía en esa buena exposición en la vertiente norte del macizo de Alcoy, donde los flujos del NE llegan aún con facilidad. Con esos rasgos, las precipitaciones se cifran en $823 \mathrm{~mm}$ en un año medio, si bien en años húmedos se llega a superar los $1.700 \mathrm{~mm}$; lo cual es indicio de las notorias oscilaciones del balance hídrico, con valores medios de $227 \mathrm{~mm}$ y máximos de $1.030 \mathrm{~mm}$ para el superávit de agua, y que se corresponden con coeficientes de escorrentía entre 0,22 y 0,55 respectivamente.

En la subcuenca del Guadalest se evidencia la reducción pluviométrica que hace descender las precipitaciones a $593 \mathrm{~mm}$ en un año medio y, a $1.079 \mathrm{~mm}$, para un año húmedo; a estos valores le corresponden superávits de agua entre $108,9 \mathrm{~mm}$ y $544,1 \mathrm{~mm}$, y coeficientes de escorrentía que van de 0,12 a 0,26 respectivamente.

Con todo, la mayor reducción pluviométrica y de conjunto de los coeficientes de escorrentía se opera en el bajo Algar-Guadalest, donde el superávit de agua es nulo en un año medio y sólo de $171,8 \mathrm{~mm}$ en el año húmedo, a lo que corresponden valores del coeficiente de escorrentía de 0,002 y 0,16 respectivamente.

En definitiva, el valor que ofrece en conjunto la cuenca oscila entre 0,14 y 0,36 para el coeficiente de escorrentía, pero queda claro que en estos valores lo decisivo son las cabeceras y, en concreto, el alto Algar, merced al acuífero del Carrascal-Ferrer, y el alto y medio Guadalest, por las surgencias del sistema de Aitana y Serrella-Xortà, capaces de regular las precipitaciones abundantes que se producen en estos sectores.

Desde un punto de vista morfológico y pluviométrico, el río Algar-Guadalest muestra dos subcuencas bien diferenciadas correspondientes a las dos ramas principales que lo forman, en las que los rasgos estructurales y litológicos deciden una morfología y morfometría peculiares.

Morfométricamente, ambas subcuencas alcanzan un desarrollo de seis según la jerarquía que establece STRAHLER (1974), lo que proporciona un orden siete para el colector 
en su desagüe. Se trata, por lo tanto, de una cuenca con una categoría fluvial bastante alta a tenor del grado de desarrollo superficial, que es relativamente discreto $\left(212,26 \mathrm{Km}^{2}\right)$; categoría fluvial que, de otra parte, queda justificada tanto por los fuertes desniveles, como por los altos coeficientes de escorrentía.

En lo concerniente al número de cauces y su longitud, se establece una clara distinción entre las dos subcuencas, puesto que son más abundantes y menos desarrollados en el Algar que en el Guadalest, en el que muestran mayor longitud y menor cantidad. En conjunto, ofrecen una densidad de avenamiento de $3,92 \mathrm{Km} / \mathrm{Km}^{2}$, que puede considerarse de grado medio si tenemos en cuenta el umbral pluviométrico de la cuenca y los desniveles (31 por mil en el cauce principal); ahora bien, el grado de permeabilidad determina que, salvo en la depresiones tectónicas de Tárbena y Guadalest, en los taludes del paleoceno de las vertientes septentrional y oriental de Aitana y en el afloramiento diapírico, el resto de la cuenca presenta unos niveles de infiltración acusados — manifiestos en algunos complejos cársticos, especialmente de Aitana- que justifican los valores de la densidad de drenaje comentados anteriormente.

Sin embargo, a efectos de avenidas, son los datos de relación de bifurcación $(3,53)$ y razón de elongación de la cuenca $(0,57)$ los que aportan mayores luces. Se trata de una cuenca de compacidad media, valor que resulta del promedio entre las dos ramas afluentes; asimismo, el valor de la relación de bifurcación es relativamente bajo, indicativo de un comportamiento sin grandes ondas de crecida. Si bien esta afirmación requiere una matización.

Obviamente, el embalse en la cabecera del río Guadalest, actúa como elemento de control de cara a un comportamiento torrencial de esta subcuenca, de manera que únicamente permanecen al margen de regulación efectiva las pequeñas cuencas situadas aguas abajo del citado embalse y la subcuenca del río Algar; dato a tener presente por cuanto que es esta subcuenca la que presenta unos coeficientes de peligrosidad mayores, por tratarse de un complejo hidrográfico con relaciones de bifurcación bajas, cuenca más compacta, fuertes desniveles y una cabecera impermeable en la que los coeficientes de escorrentía pueden llegar a ser muy altos.

\subsection{Unidades fisiográficas}

Con esta denominación identificamos aquellos aspectos que son propios de la morfología de este tipo de aparatos fluviales (vid. figura 2). En primer lugar, conviene aclarar cuáles son los límites reales del río como sistema morfológico; delimitación que viene determinada por el proceso de instalación de la red de avenamiento y, concretamente, del cauce que jerarquiza la escorrentía generada en una cuenca vertiente de considerables dimensiones dentro del contexto hidrográfico de la provincia de Alicante, 212,2 $\mathrm{Km}^{2}$.

Dicho proceso hay que relacionarlo con los fenómenos de acumulación-erosión en este tramo bajo de la cuenca; en efecto, las variaciones en la naturaleza de este sector más bajo, a partir de la confluencia entre el Algar propiamente dicho y el Guadalest, son las que, en última instancia, deciden este rasgo. Es necesario pensar en un valle estructural que determinó el sentido de la escorrentía y localización del canal de salida hacia el mar que, en una primera fase de evolución (Cuaternario antiguo), quedó definida como área de acumulación; en definitiva, de aluvionamiento. Momento en el que se estableció un nivel de circulación y depósito que, posteriormente, deviene en sector de predominio de fenómenos erosivos. Con ello, se produce el encajamiento del río en sus propios sedimentos, de manera que éstos que- 


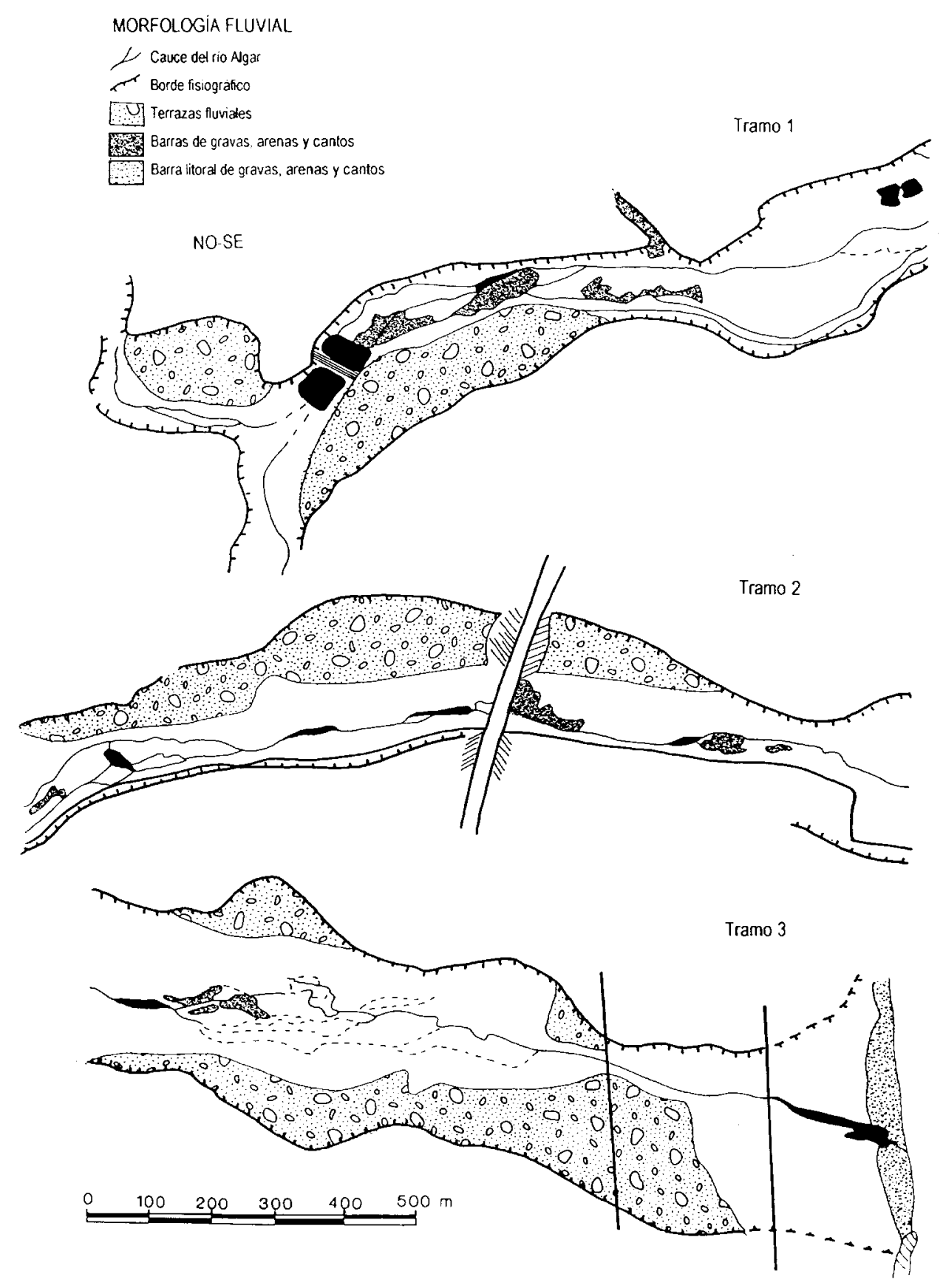

FIGURA 2. Morfología fluvial y unidades fisiográficas. 
dan colgados respecto del cuérnago del valle, es decir, pasan a ser terrazas fluviales, concretamente la más alta, que, a su vez, constituye la superficie de referencia para definir el encajamiento del río; en la actualidad se trata de los bordes fisiográficos del cauce.

Éstos, delimitan perfectamente el espacio que en la actualidad se percibe como río; no obstante, si en las inmediaciones de la confluencia con el Guadalest, las márgenes están claramente definidas, en el desarrollo longitudinal del río — como es lógico-, éstas pierden definición; puesto que se constata un progresivo descenso del desnivel entre los bordes mencionados y el talweg, de tal manera que, a partir de la N-332, aproximadamente, éstos quedan prácticamente diluidos en un amplio llano de inundación que coincide con el sector de la desembocadura, en sentido amplio.

Dentro de los límites señalados, se observa la presencia de otras unidades condignas al sistema fluvial. Obviamente, los referidos procesos de acumulación-erosión y consiguiente encajamiento del cauce, se han ido sucediendo en el tiempo, de manera que, por debajo de estos bordes, se localizan depósitos aluviales de edad más reciente, testigos de otros niveles de circulación subaérea de las aguas que, en la actualidad y por los sucesivos encajamientos, quedan configurados, así mismo, como terrazas fluviales situadas a niveles inferiores (representadas como tales en el croquis correspondiente).

En la parte más baja, se encuentra el nivel actual de acumulación aluvial que se identifica con el lecho de inundación. No obstante, hay que tener en cuenta, por un lado, las características hidrológicas de la cuenca: comportamiento espasmódico relacionado con un régimen pluvial de tipo torrencial, con acusados estiajes y períodos de sequía; y, por otro, las condiciones morfológicas de la misma: cursos cortos, próximos al mar, con acusadas pendientes, en suma, con elevada competencia y capacidad de arrastre. En definitiva, la morfología de detalle del lecho queda definida por la alternancia de barras y surcos; barras que en momentos de aguas bajas permanecen emergidas y destacadas en este contexto y, a menudo, desprovistas de vegetación; por su parte, los surcos, constituyen los sectores más bajos y, por ende, los utilizados por las aguas para discurrir, podríamos referirnos a canales de estiaje, puesto que no se trata de un lecho único; se configura como el típico lecho de cauces anastomosados o entrelazados en los que, además, por la dificultad para el fluir que supone la presencia de las barras, es habitual la existencia de remansos, incluso hoyas, coincidiendo con los surcos más acusados.

Finalmente, en la desembocadura se configura una barra de cantos, gravas y arena que, por la remodelación de la estructura sedimentaria impuesta por los sistemas de corrientes marinas y de oleaje, actúa como cierre del tramo final del Algar, dando lugar a la formalización de un área en la que se amplía el perímetro de la lámina de agua.

\subsection{Formaciones vegetales}

En una primera aproximación puede resultar paradójico el hablar de formaciones vegetales para referirse a los poblamientos vegetales del cauce de un río. No obstante, la escala de análisis empleada parecía aconsejar la diferenciación entre las distintas masas en atención a los portes alcanzados, de ahí que, en la cartografía presentada, se lleve a cabo esa individualización entre: por un lado, los sectores donde la fisonomía — no su naturalezade los elementos que las componen alcanza el porte arbustivo o arborescente de forma dominante; y, por otro, aquellas áreas en las que convergen tanto las características fisiológicas como las morfográficas en la definición de dichos poblamientos como subarbustivos o herbáceos (vid. figura 3). 

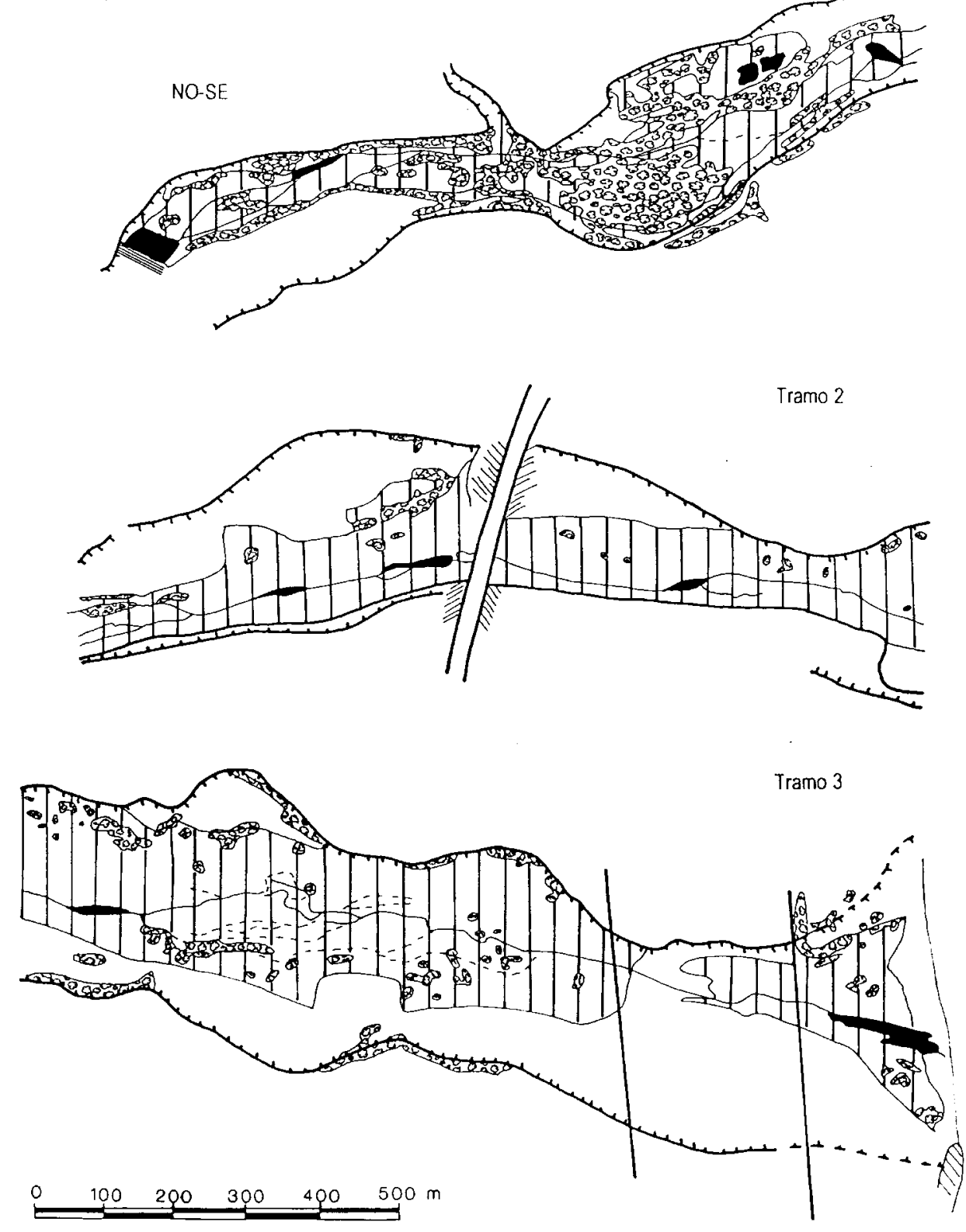

FIGURA 3. Formaciones vegetales. 
Sin embargo, paso previo a la descripción que hemos señalado, es apuntar una serie de consideraciones de carácter general. A nadie debe escapar un hecho, sobre el que se incidirá más adelante, buena parte del río, de su cauce, es percibido actualmente como un espacio natural, y esto, a pesar de la intensa humanización de la que ha sido objeto: regulación en cabecera, encauzamiento parcial, azud en el tramo considerado, extracción de aluviones para «áridos», regulaciones y sangrías en el tramo bajo; en definitiva, mecanismos que darían pie a pensar en una intensa degradación.

Por contra, el lecho del río, como ecótopo singular, presenta unas características igualmente especiales; de forma muy genérica, podemos decir que se trata de una zona húmeda, y dicha conceptuación le confiere, a su vez, un alto grado de respuesta ante tan elevado cúmulo de alteraciones. En estos espacios, la recuperación de la naturaleza es inmediata; utilizando una expresión más ajustada, se puede afirmar que los procesos de regeneración propios de estos lugares se pueden medir temporalmente a escala humana. Baste con avanzar que en 1956 el lecho del río Algar era un impresionante cauce completamente tapizado por el canturral y desprovisto de vegetación, en 1978 mostraba todos los signos y abalorios propios de un recurso geomorfológico intensamente aprovechado mediante la extracción de materiales para la construcción inmobiliaria y, en la actualidad, basta con visionar los croquis relativos a las formaciones vegetales para comprender que el cambio progresivo experimentado ha sido radicalmente positivo, incluso, se podría decir que, en algunos casos, el abandono de algunas actividades que afectaban al cauce ha sido beneficioso, por cuanto que han conferido un mayor grado de diversidad biológica (biodiversidad) a este ecótopo: caso paradigmático son las pozas y pequeñas charcas - láminas de agua - que se identifican con antiguas extracciones o pequeños encauzamientos (vid. figura 4).

Todo ello nos está dando información acerca de un ambiente sumamente dinámico, no sólo desde un punto de vista morfológico, sino, sobre todo, biogeográfico. El tapiz vegetal existente es, pues, relativamente joven y, como tal, escasamente estructurado. En efecto, si

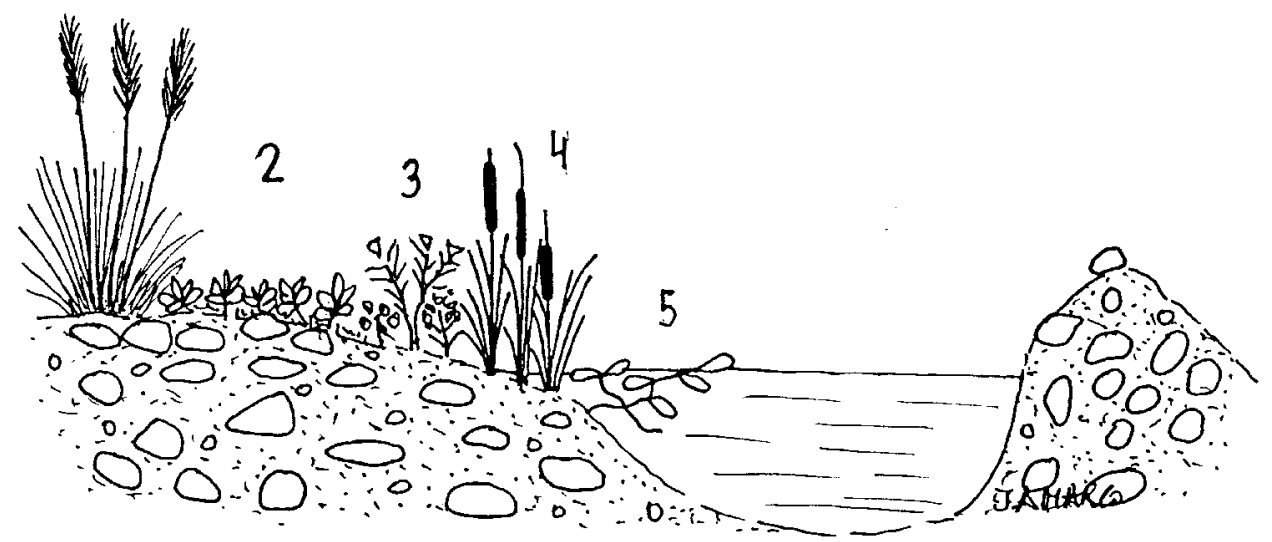

FIgURA 4. Esquema de la vegetación en una poza. 1. Sisca (Erianthus ravennae). 2. Herbazal de Potentilla reptans. 3. Adelfilla pelosa (Epilobium hirsutum), Mentha aquatica y Polygonum salicifolium. 4. Vegetación helofítica con enea (Typha angustifolia). 5. Vegetación acuática (Potamogeton sp.). 


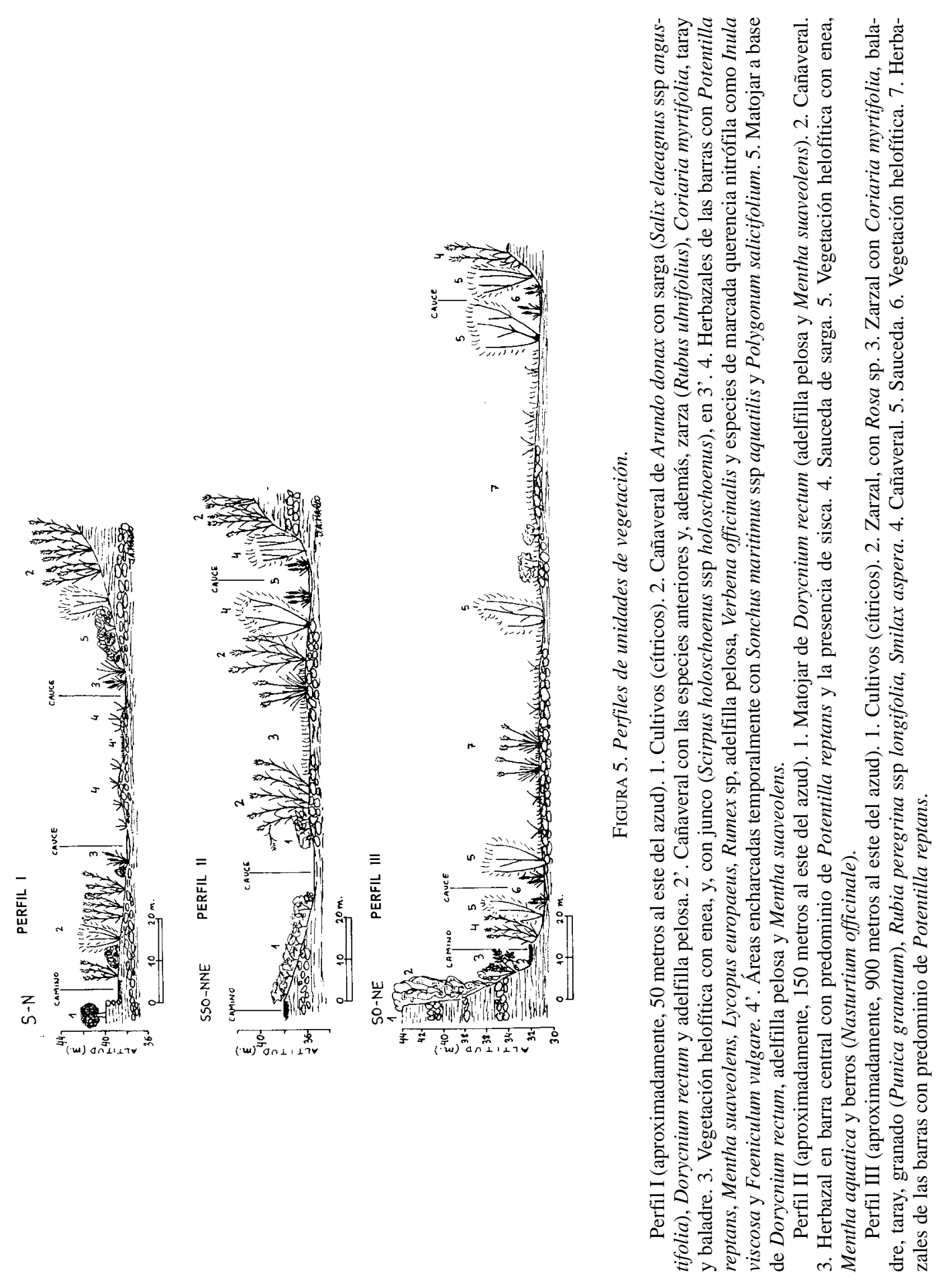




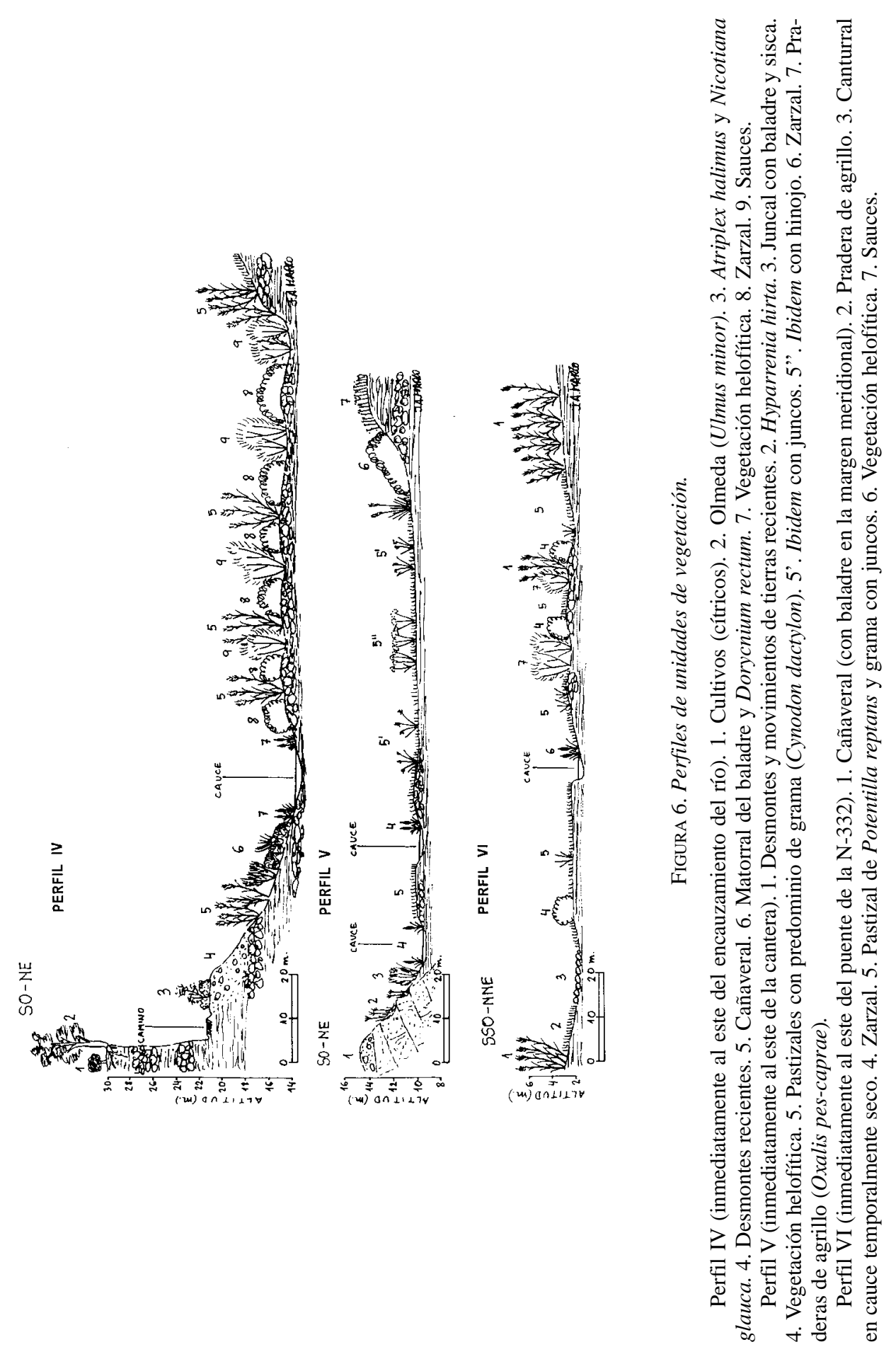


bien se esboza, no se observa con nitidez, una disposición transversal típica de la vegetación riparia; es decir, las franjas paralelas habituales en los cursos fluviales en las que las especies se sitúan en orden decreciente en exigencia hídrica desde el cauce a sus bordes.

Sin embargo, si se observa una zonación de unidades de vegetación más o menos exigentes en humedad, de manera que en las orillas más tendidas (vid. perfiles I, II y III de la figura 5), coincidiendo con remansos, aparece la típica vegetación helofítica a base de enea (Typha angustifolia), junco (Scirpus holoschoenus ssp holoschoenus), menta (Mentha aquatica) y berro (Nasturtium officinale); mientras que, en ocasiones, flanqueándola se localizan saucedas de sarga (Salix elaeagnos ssp. Angustifolia). En los bordes más externos el predominio corresponde al cañaveral (Arundo donax) con Dorycnium rectum, adelfilla pelosa, la sarga y la escandente Calistegia sepium, conjunto que se enriquece puntualmente con la presencia de Salix atrocinerea, taray, baladre (Nerium oleander) e, incluso, Rubus ulmifolius y Coriaria myrtifolia. Especies estas dos últimas, que, junto a algún rosal silvestre, rubia (Rubia peregrina ssp longifolia), zarzaparrilla (Smilax aspera), granado (Punica granatum), taray y adelfa, constituyen una maraña que se encarama en el borde vertical de la margen meridional derecha del río (vid. perfil III de la figura 5) en la que, también de forma puntual, se han localizado restos de olmeda (Ulmus minor) en la parte superior de la misma (vid. perfil IV de la figura 6).

En las áreas encharcadas temporalmente (vid. perfil 1 de la figura 5) de la parte central del cauce destacan Sonchus maritimus ssp aquatilis y Polygonum salicifolium, mientras que en las barras (vid. perfiles I, II y III de la figura 5), más altas que las anteriores, encontramos Mentha suaveolens, adelfilla pelosa (Epilobium hirsutum), Potentilla reptans, Verbena officinalis, Rumex sp, Lycopus europaeus, sisca (Erianthus ravenae) y especies de marcada querencia nitrófila como Inula viscosa e hinojo (Foeniclum vulgare).

No obstante, la progresión señalada no es uniforme en todo el desarrollo del cauce, del mismo modo que tampoco lo son las intervenciones antropogénicas en su intensidad, de manera que a lo largo del cauce pueden identificarse varias zonas, cuyo criterio de diferenciación adoptado es el porte de los poblamientos vegetales. Obviamente, los tramos donde el dominio corresponda a las formaciones arbustivas y arborescentes representan las áreas de impactos menos intensos, más alto grado de recuperación y, por consiguiente, de mayor calidad ambiental. Con estos rasgos casa perfectamente el tramo más alto del río en término municipal de Altea, es decir, desde el azud hasta prácticamente el comienzo del encauzamiento en torno al paso de la autopista. Las especies que, de manera más frecuente, constituyen dichos poblamientos son las saucedas, básicamente con el dominio de Salix elaeagnus ssp angustifolia; el cañaveral de Arundo donax con baladre, taray, Dorycnium rectum y Calistegia sepim; el zarzal en el que destacan sobre todo Rubus ulmifolius y Coriaria myrtifolia y, por último, la olmeda.

Por su parte, los poblamientos caracterizados como subarbustivos y herbáceos, se intercalan entre estos pequeños bosquetes, bien porque se trate de sectores en los que destaque la presencia de barras de cantos y gravas, bien porque aparecen algunos remansos en los que como se ha podido comprobar en los perfiles realizados, sí se esboza una estructuración transversal al sentido de la corriente, de manera que, en contacto directo con el agua —vegetación helofítica-, el dominio corresponde a la enea (Thypha angustifolia), situación que se repite en varias ocasiones a lo largo de este primer tramo; además, también son frecuentes la Mentha aquatica, el berro (Nasturtium officinale), Sonchus maritimus ssp aquatilis o Polygonum salicifolium. En los herbazales de las barras o crestas del cauce, además de algunas herbáceas vivaces (Erianthus ravenae), destacan poblamientos casi especí- 


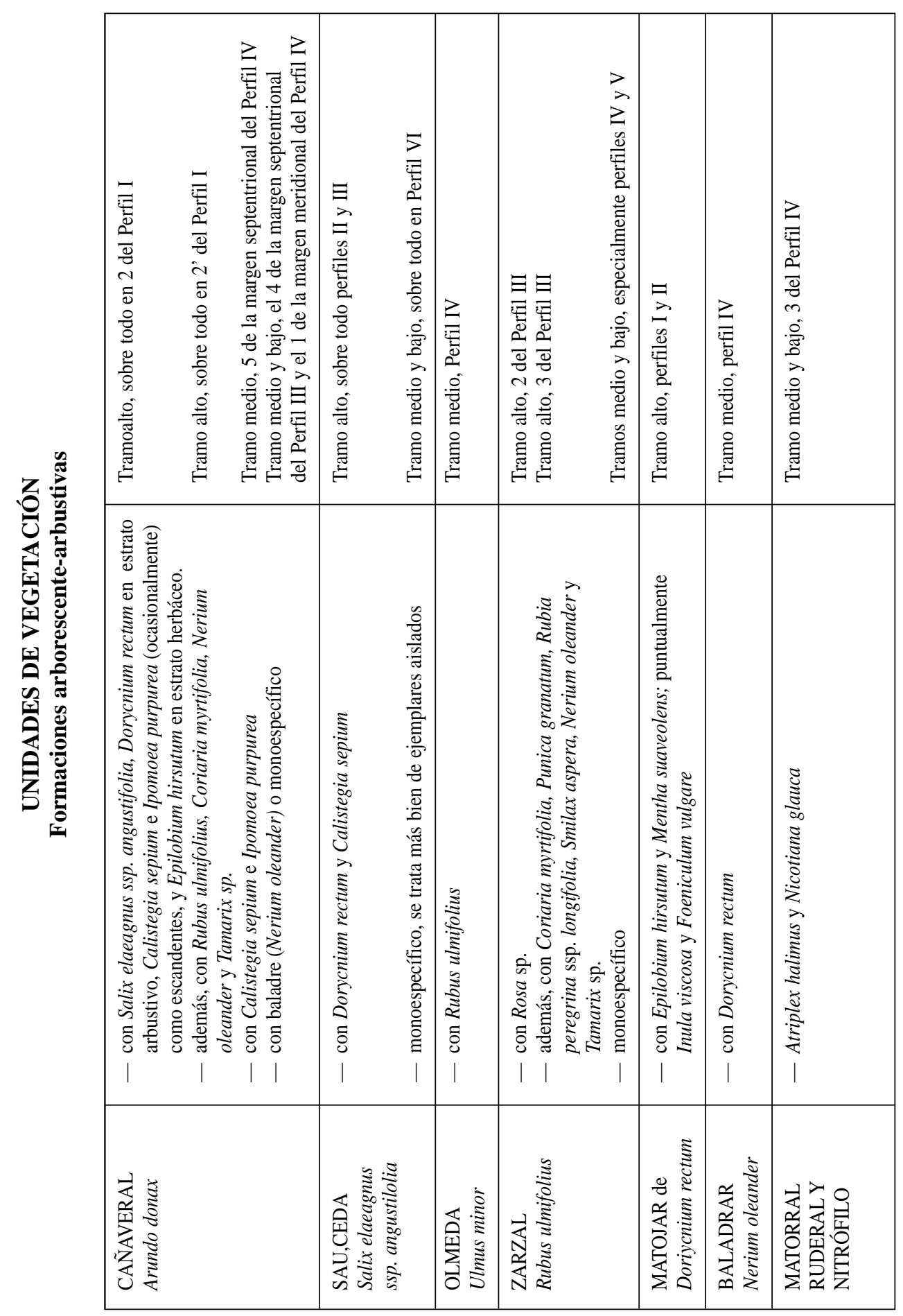




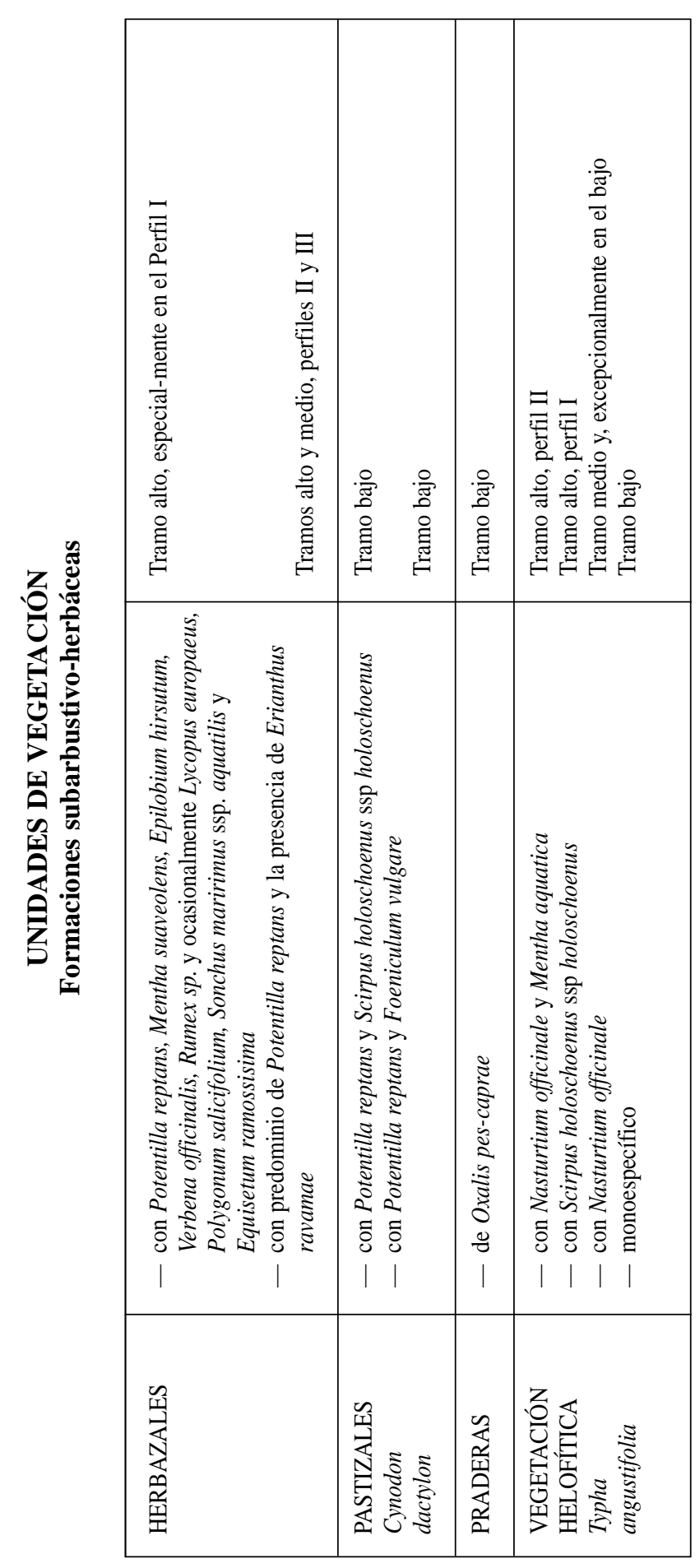


ficos de Potentilla reptans y grama (Cynodon dactylon) con Rumex sp, la adelfilla pelosa, Mentha suaveolens, entre otras.

A estas especies subarbustivas y herbáceas señaladas anteriormente corresponde el predominio en el segundo tramo reconocido, cuyas dimensiones coinciden con las del encauzamiento del río a ambos lados del paso de la autopista; el dominio de este tipo de poblamientos es casi absoluto, reflejo de una regeneración desacelerada en relación con las obras de acondicionamiento del cauce.

Menos acentuado es el dominio señalado en el tramo siguiente que se extiende hasta la propia desembocadura, a pesar de que se trata del sector en el que las extracciones para áridos, detectadas en los fotogramas de 1978, alcanzaban mayor desarrollo. En este sector, si bien hay restos de poblamientos de carácter arbustivo o arborescente (cañaveral, zarzal y sauceda), el predominio corresponde a los poblamientos herbáceos contituidos básicamente por céspedes o praderas de grama y Potentilla reptans (vid. perfiles V y VI de la figura 6). Sin embargo, es preciso indicar que el sector comprendido entre el puente del ferrocarril y el de la carretera nacional, es el más degradado de todo el entorno, puesto que las obras de aplanamiento y movimiento de tierras recientes han interrumpido el ritmo de regeneración de la cubierta.

\subsection{Intervenciones antropogénicas}

Las condiciones topoecológicas que se acaban de detallar han merecido una valoración muy variable a lo largo de la historia; valoración que, en definitiva, ha marcado las pautas en la ocupación y aprovechamiento de un ecótopo de estas características. Como referencia diacrónica obligada, se debe pensar en la profunda transformación que supone el paso de una economía agraria, prácticamente autárquica, hacia una sociedad encaminada a una terciarización que define las coordenadas económicas actuales.

Esa disparidad conceptual implica una percepción igualmente contrastada del espacio analizado; basta con señalar cuáles eran las pautas de la organización tradicional del espacio hasta mediados del siglo actual, para comprobar hasta que punto dicha transformación supone la aparición de actividades cuyas consecuencias y efectos medioambientales escapan al simple calificativo de intervenciones o aprovechamientos, y casan perfectamente con lo que podría conceptuarse como «impacto» (con sus diferentes cargas peyorativas).

Efectivamente, las labores de fotointerpretación llevadas a cabo con los fotogramas correspondientes a 1956, revelan la existencia de acusadas diferencias en relación a las imágenes de 1992. Así, en aquella época es visible que los aprovechamientos propios de las unidades de este ecótopo que presentan cierta peligrosidad, son los relacionados únicamente con la actividad agraria predominante; es decir, a lo sumo, las terrazas están ocupadas por campos de cultivo, mientras que el lecho de inundación permanece al margen de cualquier ocupación de carácter permanente, de manera que sólo se aprovecha como lugar de aprovisionamiento de ciertos materiales — cañas—, de tal modo que aparecía casi desprovisto de vegetación, es decir, aflorando directamente el canturral condigno a un lecho de estas características.

De esta organización esquematizada perduran en el paisaje actual, sobre todo, las vinculadas a la actividad agraria, localizada predominantemente en las terrazas medias y bajas del río, mientras que otras, intensas antaño, han disminuido en intensidad. Las necesidades de la sociedad actual han determinado la aparición de nuevos aprovechamientos y ocupaciones del territorio; progreso y mejora en la «calidad de vida» quedan evidenciados en una serie de actuaciones de considerable impronta paisajística; posiblemente sea, en lo concer- 
- INTERVENCIONES ANTROPOGÉNICAS

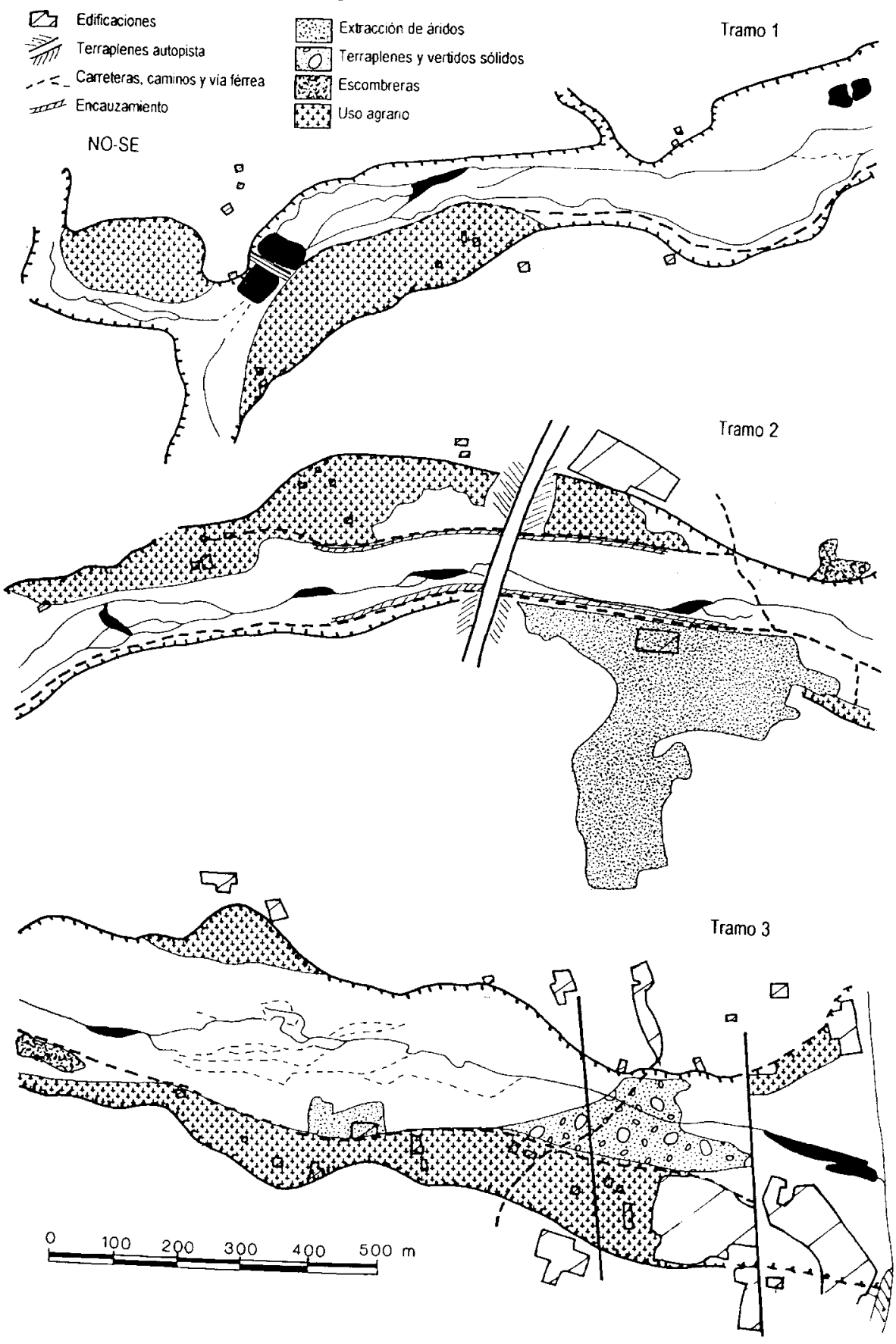

FIGURA 7. Intervenciones antropogénicas. 
niente a las infraestructuras, uno de los aspectos en los que mejor se plasmen las transformaciones a las que se ha hecho referencia con anterioridad (vid. figura 7). Además de la aparición de viales secundarios, tanto en sentido transversal como longitudinal al cauce, destacan, no obstante, los tres ejes que, de forma transversal, atraviesan el río: carretera nacional 332, ferrocarril de vía estrecha Alicante-Denia y, sobre todo, el trazado de la autopista del Mediterráneo (A-7, E-15). Con todo, es esta última, la que acapara de forma más evidente el protagonismo como elemento de alteración y elevada impronta — podría decir también impacto- en el paisaje y el medio, tanto por las obras de terraplenado a ambos lados del río, como por el encauzamiento y artificialización del lecho del mismo.

Por otra parte, el emplazamiento de viviendas y equipamientos, son otro de los elementos a considerar. Si bien, es necesario señalar que la mayor parte de las edificaciones de carácter permanente aparecen dispersas por la mayor parte de las terrazas y de forma puntual, es decir, perfectamente imbricadas en la trama del paisaje agrario; otras, en cambio, por lo notorio de sus volúmenes o superficies afectadas representan un impacto, no sólo de tipo perceptivo, sino también medio ambiental; tal es el caso de las instalaciones deportivas ubicadas en la margen derecha del tramo final del río y la urbanización situada entre la carretera nacional y la línea de costa en la misma margen.

Tratamiento aparte merecen otras actuaciones, como son las actividades extractivas bien por la proximidad del afloramiento de las calizas que se aprovechan para áridos de la cantera situada inmediatamente al este del paso de la autopista, bien porque se aprovechen los materiales aluviales del río, como queda evidenciado en la actualidad en la instalación localizada unos 400 metros aguas arriba del paso del ferrocarril; en cualquier caso se trata de un serio impacto materializado tanto por las obras de terraplenado, como por las labores de excavación llevadas a cabo. Afortunadamente, esta última actividad, más controlada por la legislación vigente, está concentrada en el punto indicado, a diferencia de lo que parecía norma común en otros momentos, especialmente, a finales de la década de los setenta, cuando las necesidades de materia prima derivadas de un acelerado proceso urbanizador, generaron un aprovechamiento generalizado de gran parte del cauce y de las terrazas, de tal modo que se produjo una intensa degradación del fondo del lecho. No obstante, la respuesta de la naturaleza tras el abandono de estas actividades, ha sido inmediata, a la vez que, alguno de los vaciados generados por aquéllas y, posteriormente inundados por las aguas del río, son motivo de aparición de zonas encharcadas y, por ende, han conferido una mayor biodiversidad a este espacio.

Por último, es preciso referirse a dos hechos que adquieren cierta importancia en la definición del estado actual de parte del cauce: en un caso se trata de señalar los posibles efectos negativos del vertido de las aguas de la depuradora al lecho del río, aguas abajo del paso de la A-7, así como la constatación de un aprovechamiento ganadero, de raigambre tradicional, que afecta, sobre todo, al tramo más bajo del río. Hechos que convergen con los señalados más arriba y que justifican la mayor degradación de esta zona respecto de los espacios más próximos al azud.

\section{Fase sintética o prospectiva}

\subsection{Riesgos relacionados con el río y unidades fisiográficas}

Posiblemente sea el de «riesgo natural» uno de los conceptos que implique de forma más directa la evidente interacción entre el hombre y la naturaleza, interacción que com- 
prende relaciones en ambos sentidos, es decir, tanto de lucha y adaptación del hombre a unas condiciones determinadas, como de incapacidad de controlar ciertas situaciones que, a veces, pueden resultar catastróficas. En el presente estudio se pretende poner de manifiesto tales interrelaciones, especialmente con una clara referencia a la ordenación del territorio.

Es por ello, que se haga necesaria una rigurosa valoración de los recursos geomorfológicos del espacio analizado, al objeto de definir tanto la peligrosidad inherente a cada una de las formas de modelado señaladas, como el riesgo derivado de los impactos provocados por la intervención o posibles intervenciones antrópicas. La identificación y conceptuación de las distintas unidades morfológicas que se articulan en el ámbito estudiado, así como los fenómenos condignos a cada una de dichas unidades morfológicas, son claves para la prevención de los posibles problemas que puedan derivar de los mismos.

Estos últimos son los que se identifican con la peligrosidad inherente a cada uno de ellos, así como con el riesgo derivado de las actuaciones antropogénicas -impactosplanteamiento que viene a matizar el propuesto por PANIZZA (1990). Una vez concluido este análisis general, el objetivo del trabajo se centra en la valoración específica de cada una de estas unidades con el fin de determinanr la capacidad de acogida del territorio, teniendo en cuenta que, a su vez, cada una de estas unidades presenta cierta vulnerabilidad ante determinadas actuaciones, así como un cierto grado de peligrosidad. Se trata, en definitiva, de efectuar un diagnóstico valorativo del medio físico que permita realizar una caracterización de los procesos que potencialmente impliquen mayor riesgo. En este sentido, cabe indicar que el desarrollo correcto de este esquema tiene como pilar fundamenta el conocimiento de las relaciones existentes entre determinadas actuaciones antropogénicas y los mecanismos morfológicos; es decir, sólo si conocemos los efectos de las primeras, podremos señalar que tipo de medidas correctoras deben ser consideradas, o bien, que actividades deben limitarse.

Realizada la caracterización y conceptuación de las distintas formas ligadas al sistema fluvial (vid. figura 2), es obligado referirse al grado de peligrosidad que muestra cada una de ellas, así como la vulnerabilidad relacionada con ciertas intervenciones antropogénicas. Obviamente, tanto un aspecto como el otro son deudores del reconocimiento de los procesos o fenómenos que tienen lugar en estos recursos geomorfológicos que constituyen este sistema fluvial.

En este sentido, las formas identificadas que merecen especial atención es el propio lecho, y las terrazas fluviales ligadas al mismo. En un esquema morfológico de la red de avenamiento, el primero, es decir, el cauce, es la unidad en la que se generan y formalizan las ondas de crecida en las avenidas y riadas; como quiera que el encajamiento del cauce disminuye paulatinamente desde su cabecera hacia la desembocadura, los caudales vehiculados experimentan igualmente una alteración en este mismo sentido, puesto que se pasa de sectores en los que la sección transversal del cauce está perfectamente definida, a otros, en los que dichos límites se diluyen y casi terminan por desaparecer, de manera que se pasaría de un cauce propiamente dicho a un llano de inundación, o en el caso que nos ocupa, a un lecho de inundación en las inmediaciones de la desembocadura. La actualización o puesta en valor de la peligrosidad reseñada, deriva de la ocupación de dichas unidades morfológicas, e incluso, de la modificación del primitivo cauce.

En cualquier caso, estos procesos adquieren consecuencias más importantes debido a otros factores, tanto de índole natural, como relacionados con intervenciones antropogénicas. En el primero de los supuestos, cabe citar el efecto de tapón para el desagüe que supone el aumento momentáneo del nivel marino por la coincidencia de los procesos de avenida 
con flujos de levante, así como la presencia de la citada barra de cantos en la línea de desembocadura del río Algar, que contribuye a dificultar la libre circulación hacia el mar, de forma que se genera un dañino aumento de los calados de las aguas de avenida y la consiguiente inundación de los espacios aledaños al tramo más bajo del río; de hecho, los episodios de lluvias torrenciales más recientes —especialmente el de 1986—, pusieron de manifiesto que la zona del polideportivo y urbanizaciones contiguas — situadas en el lecho de inundación - son las áreas de mayor riesgo de avenida e inundación del mismo.

Menos dañinos y llamativos son los fenómenos que se relacionan con los sistemas de terrazas fluviales y márgenes del cauce, donde los procesos dominantes derivan de los mecanismos de zapa lateral determinantes de deslizamientos y desprendimientos (solsides), reflejados en timbes, y colapsos de los conductos de circulación subsuperficial; así como surcos y regueros que surgen en el borde de las terrazas como consecuencia de la circulación subaérea de los flujos que se generan en dichas unidades. Todo ello, sin olvidar que, en última instancia, la raigambre de estos procesos se relaciona con la naturaleza detrítica de las formaciones superficiales que singularizan estas unidades.

\subsection{Zonificación de calidad ambiental}

La superposición de la información cartográfica básica obtenida mediante el análisis de morfología fluvial, formaciones vegetales e intervenciones antropogénicas, junto a la consideración de la limitación que implica la peligrosidad y riesgo del sistema fluvial indicados en el apartado anterior, ya en esta fase sintética, permite calibrar el grado de calidad ambiental del río Algar en su tramo alteano (vid. figura 8). Para ello se han diferenciado tres categorías a partir de una serie de criterios entre los que destacan:

— peligrosidad de la dinámica fluvial

- grado de cobertura de la vegetación

— porte y estructuración de las unidades de vegetación

-intensidad de las intervenciones antropogénicas

— permanencia de los efectos de dichas intervenciones

La primera de las categorías se identifica con las zonas de calidad media-alta que corresponde a aquellos sectores o tramos en los que se aprecia una situación próxima al óptimo, no porque éste se haya alcanzado de forma efectiva, sino porque se observa un ritmo de recuperación más acusado, de manera que se estima conveniente dar curso a los procesos y dinámicas naturales. No obstante, dentro de las áreas incluidas en esta categoría, es el tramo más alto el que presenta una situación más próxima a dicho óptimo; es, pues, la zona en la que mayor celo deben alcanzar las medidas de protección, a las cuales únicamente acompañarían las derivadas de regeneraciones puntuales del medio y actividades de tipo científico-didáctico. Por su parte, tanto el tramo de esta misma categoría, comprendido entre el encauzamiento del puente de la autopista y el puente del ferrocarril, como el más cercano a la desembocadura, además de las actividades indicadas en el sector anterior, pueden ser utilizados igualmente como espacios en los que tengan cabida actividades relacionadas con el ocio, el esparcimiento, y el deporte, entre otras.

Con la caracterización de calidad media, se engloban aquellas áreas en las que la permanencia de los efectos de las intervenciones antrópicas, ha desacelerado el ritmo de rege- 


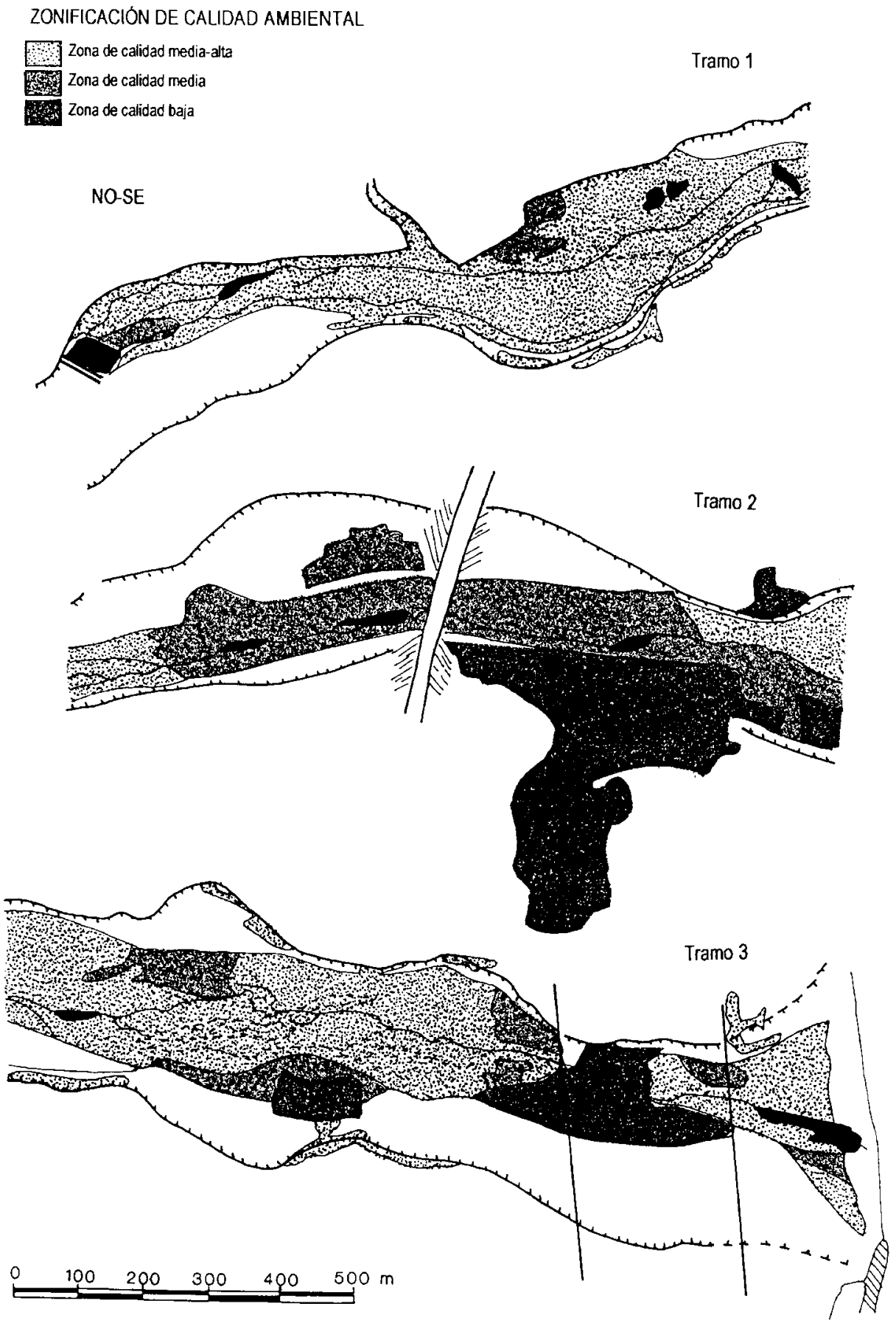

FIGURA 8. Zonificación de calidad ambiental. 
neración de los poblamientos vegetales. Se trata de sectores que es preciso recuperar y elevar el nivel específico de calidad ambiental hasta igualarse con los sectores de la categoría precedente con los que limitan. Para ello, es necesario realizar labores de restauración ambiental que cumplan dichos fines, de manera que, cuando éstos se hayan alcanzado, pasen a regirse por los principios de gestión señalados para cada uno de los tramos indicados anteriormente, si bien, los más restrictivos tendrían como límite más oriental, el inicio del encauzamiento del río al paso de la autopista.

Por último, el nivel más bajo de calidad ambiental corresponde a aquellas zonas en las que, tanto la intensidad, como la permanencia de las intervenciones antropogénicas alcanzan su grado máximo, dificultando, en gran medida, la recuperación del ecótopo que caracteriza a las categorías anteriores, pese a que en alguna ocasión desborda los límites propios del espacio analizado — cantera inmediatamente al este de la autopista—; razón por la que parece aconsejable dedicar estos espacios a la instalación de posibles infraestructuras y edificaciones permanentes relacionadas con las actividades propuestas en las categorías anteriores (aula de la naturaleza, centro de acogida de visitantes...).

Todas estas medidas generales referidas a las tres zonas de calidad ambiental diferenciadas, deberían acompañarse de otras de carácter sectorial encaminadas a potenciar la regeneración o mantenimiento de las condiciones ecológicas óptimas. Entre otras, es aconsejable el tratamiento integral de los caudales del río; ya que, en la actualidad, las aguas «depuradas» vierten nuevamente al cauce, aguas abajo de la autopista. Tanto por su calidad como por el volumen evacuado merecen ser reconducidas; pues, una mejora en la depuración de las mismas disminuiría los posibles efectos negativos que éstas puedan tener sobre fauna y flora; de manera que, mediante una elevación de las mismas hasta el azud podría utilizarse como «caudal ecológico», especialmente en los años de mayor escasez de precipitaciones.

En resumen, esta zonación y las indicaciones puntuales realizadas en cada una de las unidades, pueden ser la pauta a seguir de cara a abordar la ejecución de un plan de uso y gestión — ya a escala de diseño-, tendente a la formalización de un futuro PARQUE

\section{FLUVIAL DEL RÍO ALGAR.}

\section{Bibliografía}

CEOTMA (1984): Guía para la elaboración de estudios del medio físico. MOPU, Madrid. GÓMEZ OREA, D. (1994): Ordenación del territorio. Una aproximación desde el Medio Físico. ITGE, Madrid.

GONZÁLEZ BERNÁLDEZ, F. (1989): «La integración forzada de la ecología en los estudios urbanos y regionales», Ciudad y Territorio, 81-82, Madrid. Pp. 92-97.

McHARGH, I.L. (1969): Design with nature, The Natural History Press, New York.

PANIZZA, M. (1990): «Geomorfologia Applicata al rischio e all'impatto ambientali. Un esempio nelle Dolomiti (Italia)», I Reunión Nacional de Geomorfología, 17-20 septiembre 1990, Instituto de Estudios Turolenses-Sociedad Española de Geomorfología, Teruel.

STRALHER, A. (1974): Geografía Física, Omega, Barcelona. 